\title{
(3) Collection of skin biopsies from cattle
}

\section{UNIVERSITY BOVINE EI}

OCT 07, 2020

\section{open $\boldsymbol{\text { Access }}$}

\section{DOI:}

dx.doi.org/10.17504/protocol s.io.bm3yk8pw

\section{Protocol Citation: Froylan} Sosa, Peter J Hansen 2020. Collection of skin biopsies from cattle. protocols.io https://dx.doi.org/10.17504/p rotocols.io.bm3yk8pw

License: This is an open access protocol distributed under the terms of the Creative Commons Attribution License, which permits unrestricted use, distribution, and reproduction in any medium, provided the original author and source are credited

\section{Protocol status: Working} We use this protocol and it's working

\section{Created: Oct 07, 2020}

Last Modified: Oct 07, 2020

\section{PROTOCOL integer ID:} 42840

Keywords: biopsy, skin

\section{Peter J}

Froylan Sosa ${ }^{1}, \quad$ Hansen $^{1}$

${ }^{1}$ University of Florida

Peter J Hansen

University of Florida

\section{ABSTRACT}

This is a simple protocol for collecting skin biopsies from cattle. The procedure described here was used to collect skin for histological analysis but it could also be used to collect skin for PCR or other purposes. Our experience indicates that there are few complications with the procedure. The most common is that occasionally there is a slight amount of bleeding that can be treated by applying pressure using a gauze. Healing occurs rapidly and biopsy sites are difficult to detect 14 days after the procedure. The protocol is best read by using the attached pdf file.

\section{ATTACHMENTS}

$$
\begin{aligned}
& \text { Collection of skin } \\
& \text { biopsies from cattle Sosa } \\
& \text { and Hansen } \\
& \text { 10.06.2020.pdf }
\end{aligned}
$$

\section{MATERIALS}

\section{Hair clippers}

\section{Non-sterile gauze}

$3.70 \%$ ethanol-soaked gauze (made with non-sterile gauze)

4. Sterile gauze

5.5\% Lidocaine (spray). Examples include Aspercreme with Lidocaine Dry Spry and Extra

Strength Numbify - 5\% Lidocaine Numbing Spray.

6.Integra Miltex $5 \mathrm{~mm}$ standard biopsy punch - Integra cat no 3335 and Fisher cat no $12-460-411$

7.Super glue (for example, 15 gram bottle of Gorilla Super Glue Gel)

8.Adison thumb forceps or 25-gauge needle

9. A container with $10 \%$ neutral buffered formalin (NBF)

10. Tissue cassettes with two sponges (for example, Kartell, Cat 2922-04)

11. Prozap Screw Worm and Ear Tick Aerosol (Neogen)

\section{Procedure}


1 Restrain the animal in a head chute.

2 Label the cassettes - make sure to use a permanent marker for histology or pencil.

3 Clip the biopsy area with clippers and wipe down with ethanol-soaked gauze. We typically sample the thorax (middle of 5 or 6 th rib) - or shoulder area

$4 \quad$ Wipe area with a sterile dry gauze.

$5 \quad$ Spray $5 \%$ lidocaine onto the biopsy area and wait for $30 \mathrm{sec}$.

6 Collect skin sample by rotating the $5 \mathrm{~mm}$ skin punch while pressing downwards with mild force.

7 Clean the skin punch by immersing in $70 \%$ ethanol. The skin punch can be used three to four times.

8 Take the skin sample from skin punch with a needle or tweezers, and place it into a cassette between the sponges. Close the cassettes.

9 Place the cassette into the container with 10\% NBF at room temperature. 
10 Place sterile gauze on the biopsy site to remove excess blood or stop bleeding.

11 Place some super glue into the wound site. Wait 10 seconds.

12 Spray tick spray around the biopsy site.

13 To process the biopsy, leave the skin samples in NBF for 16-20 h at room temperature. Rinse the skin-containing cassettes in PBS twice, 15 min each time. Cut the skin samples in half and place them back into the cassette (use gloves). Store cassettes in a container containing PBS. 